\title{
Dynamique des systèmes désordonnés
}

\author{
R.M. Pick \\ Laboratoire des Milieux Désordonnés et Hétérogènes, Case 86, \\ Université Pierre et Marie Curie, 4 place Jussieu, 75230 Paris cedex 05, France
}

\begin{abstract}
Résumé: Ce bref exposé résume quelques unes de nos connaissances sur la dynamique des systèmes désordonnés, en passant successivement de l'état solide aux cristaux à désordre dynamique d'orientation puis aux liquides, en se limitant presque exclusivement aux liquides surfondus. Une description analytique n'est donnée que pour les solides monoatomiques désordonnés, le reste de la présentation étant qualitatif, quelques exemples illustrant des aspects de cette dynamique plus particulièrement liés à l'esprit de ces Journées de Neutronique
\end{abstract}

\section{INTRODUCTION}

Les pages qui suivent ne représentent ni un article de revue sur la dynamique des systèmes désordonnés, (il faudrait y consacrer un volume entier) ni même le résumé d'une conférence d'introduction, tel qu'il en est parfois donné dans certains congrès internationaux et qui permettent de faire le point sur un sujet donné. Je ne présente que des notes de cours qui ont été conçues pour permettre aux participants des 1 lièmes JDN de mieux profiter des exposés beaucoup plus précis qui ont été donnés à la suite de celui-ci. Je me suis efforcé de mettre en lumière la généralité de certains résultats et de n'introduire que petit à petit la complexité inhérente à l'étude de certains matériaux.

Dans cet esprit, la première partie s'intéresse à la dynamique des solides désordonnés (désordre purement statique) où le désordre est considéré comme une perturbation, plus ou moins importante, du solide ordonné; là encore, je passerai du plus simple, le solide monoatomique, tel qu'il peut être obtenu par la trempe ultra rapide d'un liquide métallique (verre métallique), au solide moléculaire désordonné, obtenu lui aussi par trempe, et enfin au cas plus complexe des verres iono moléculaires. Dans une deuxième partie, j'introduirai un premier type de désordre qui est celui des phases dites "plastiques" ou "réorientationelles" de certains cristaux moléculaires; dans celles-ci, chaque molécule peut avoir plusieurs orientations distinctes qu'elle explore successivement. J'évoquerai enfin très brièvement le cas du désordre dynamique de position, tel qu'il peut exister dans les liquides simples ou moléculaires; je focaliserai l'exposé sur le cas des liquides surfondus, qui peuvent parfois, par refroidissement, conduire à des verres car, dans ce cas, le découplage entre les temps caractéristiques des mouvements habituels de vibration et ceux relatifs aux mouvements de diffusion des centres de masse permet une étude plus fine de ces derniers.

J'ai gardé, le plus souvent, le style peu académique des notes primitives, y ajoutant seulement une partie des commentaires oraux qui les accompagnaient. Il en résulte un aspect un peu décousu dont je demande par avance aux lecteurs de m'excuser. 


\section{LES SOLIDES DESORDONNES}

\subsection{Le Solide monoatomique}

\section{1. 1 Formalisme Général}

Soit $\vec{R}_{\vec{L}}$ la position d'équilibre de l'atome et $\vec{U}_{\vec{L}}=U_{\vec{L}^{\alpha}}^{\alpha} \hat{e}^{\alpha}$ son déplacement (harmonique) hors de sa position d'équilibre. Si $\overrightarrow{\mathrm{r}}_{\overrightarrow{\mathrm{L}}}=\overrightarrow{\mathrm{R}}_{\overrightarrow{\mathrm{L}}}+\overrightarrow{\mathrm{U}}_{\overrightarrow{\mathrm{L}}}$ et si $\mathrm{V}\left(\overrightarrow{\mathrm{r}}_{\overrightarrow{\mathrm{L}}}, \overrightarrow{\mathrm{r}}_{\mathrm{L}^{\prime}}\right)$ est le potentiel d'interaction entre les

atomes $\overrightarrow{\mathrm{L}}$ et $\overrightarrow{\mathrm{L}}^{\prime}$, lorsqu'ils sont respectivement en $\overrightarrow{\mathrm{r}}_{\overrightarrow{\mathrm{L}}}$ et $\overrightarrow{\mathrm{r}}_{\overrightarrow{\mathrm{L}}^{\prime}}$, l'énergie totale du solide est:

$$
\mathrm{E}=\frac{1}{2} \sum_{\overrightarrow{\mathrm{L}}, \overline{\mathrm{L}}^{\prime}} \mathrm{V}\left(\overrightarrow{\mathrm{r}}_{\overrightarrow{\mathrm{L}}^{\prime}}, \overrightarrow{\mathrm{r}}_{\overrightarrow{\mathrm{L}}^{\prime}}\right)
$$

Si le symbole $\frac{\partial}{\partial \mathrm{R}^{\alpha}}$ représente la dérivée d'une fonction par rapport à la variable $\mathrm{r}_{\overline{\mathrm{L}}}^{\alpha}$ lorsque $\overrightarrow{\mathrm{U}}_{\overrightarrow{\mathrm{L}}}=0$, et $\overrightarrow{\mathrm{F}}_{\overrightarrow{\mathrm{L}}}$ la force qui s'exerce sur $\overrightarrow{\mathrm{L}}$ lorsque l'atome est en $\overrightarrow{\mathrm{r}}_{\overrightarrow{\mathrm{L}}}$, on a, au premier ordre en $\overrightarrow{\mathrm{U}}_{\overrightarrow{\mathrm{L}}}$ :

$$
\mathrm{F}_{\overrightarrow{\mathrm{L}}}^{\alpha}=-\frac{\partial \mathrm{E}}{\partial \mathrm{R}_{\overrightarrow{\mathrm{L}}}^{\alpha}}-\frac{\partial^{2} \mathrm{~V}}{\partial \mathrm{R}_{\mathrm{L}^{\alpha}}^{\alpha} \partial \mathrm{R}_{\overline{\mathrm{L}}^{\prime}}^{\beta}} \overrightarrow{\mathrm{U}}_{\overline{\mathrm{L}}^{\prime}}^{\beta}-\frac{\partial^{2} \mathrm{E}}{\partial \mathrm{R}_{\overline{\mathrm{L}}}^{\alpha} \partial \mathrm{R}_{\overrightarrow{\mathrm{L}}^{\beta}}^{\beta}} \mathrm{U}_{\overrightarrow{\mathrm{L}}}^{\beta}
$$

où le deuxième terme du membre de droite représente la force créée par les déplacements de tous les atomes autres que $\overrightarrow{\mathrm{L}}$ et le dernier terme est celle due au déplacement de l'atome $\overrightarrow{\mathrm{L}}$. Comme le premier terme est nul ( $\vec{R}_{\vec{L}}$ est la position d'équilibre de l'atome), on peut réécrire (2) sous la forme:

$$
\mathrm{F}_{\overline{\mathrm{L}}}^{\alpha}=-\widetilde{\mathrm{C}}_{\overline{\mathrm{L}}^{\prime}, \mathrm{L}^{\prime}}^{\alpha \beta} \mathrm{U}_{\overline{\mathrm{L}}^{\prime}}^{\beta}-\widetilde{\mathrm{C}}_{\overline{\mathrm{L}}, \overline{\mathrm{L}}}^{\alpha \beta} \mathrm{U}_{\mathrm{L}}^{\beta} \equiv-M D_{\overline{\mathrm{L}}, \mathrm{L}^{\prime}}^{\alpha \beta} U_{\overline{\mathrm{L}}^{\prime}}^{\beta}=M \ddot{U}_{\overline{\mathrm{L}}^{\prime}}^{\alpha} .
$$

où on a tenu compte de l'équation fondamentale de la dynamique, le terme $\overrightarrow{\mathrm{L}}^{\prime}=\overrightarrow{\mathrm{L}}$ étant contenu dans la matrice $\mathbf{D}$. On en déduit par transformée de Fourier en temps la Matrice Dynamique:

$$
\left(D_{\vec{L}^{\prime}, \vec{L}^{\prime}}^{\alpha, \beta}-\omega^{2} \delta^{\alpha, \beta} \delta_{\overrightarrow{\mathrm{L}}, \overrightarrow{\mathrm{L}}^{\prime}}\right) \mathrm{U}_{\overrightarrow{\mathrm{L}}^{\prime}}^{\beta}=0
$$

Remarques

$$
\forall \alpha, \beta \quad \sum_{\overrightarrow{\mathrm{L}}^{\prime}} \mathrm{D}_{\mathrm{L}^{2}, \overline{\mathrm{L}}^{\prime}}^{\alpha, \beta}=0 ;
$$

c'est l'invariance par translation; $\overrightarrow{\mathrm{F}}_{\overrightarrow{\mathrm{L}}}$ est nul si on déplace tout le solide de $\overrightarrow{\mathrm{U}}$.

- $D_{\vec{L}, \vec{L}^{\prime}}^{\alpha, \beta}$ est une fonction de $\vec{R}_{\vec{L}^{\prime}}-\vec{R}_{\vec{L}^{\prime}}$ dans un cristal, une fonction de $\vec{R}_{\vec{L}^{\prime}}$ et $\vec{R}_{\vec{L}^{\prime}}$ dans un système désordonné

\section{1. 2 Rappel sur le cas ordonné}

Pour trouver les valeurs et vecteurs propres de la matrice dynamique, on fait un changement de base, de la base $\overrightarrow{\mathrm{L}}$ (position d'équilibre des atomes) à la base $\vec{q}$ (espace réciproque), avec la matrice de changement de base $\left\{\mathrm{e}^{\mathrm{i} \mathrm{i} \cdot \overrightarrow{\mathrm{R}}_{\overrightarrow{\mathrm{L}}}}\right\}$. On obtient: 


$$
\begin{aligned}
& \mathrm{U}_{\overrightarrow{\mathrm{q}}}^{\alpha}=\mathrm{e}^{\mathrm{i} \mathrm{q} \cdot \vec{R}_{\hat{\mathrm{L}}}} \mathrm{U}_{\breve{\mathrm{L}}}^{\alpha}
\end{aligned}
$$

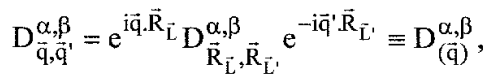

la matrice $\mathbf{D}$ ne dépendant que de $\vec{q}$ parce que, dans l'espace direct, elle ne dépend que de $\overrightarrow{\mathrm{R}}_{\overrightarrow{\mathrm{L}}}-\overrightarrow{\mathrm{R}}_{\mathrm{L}^{\prime}}$. On déduit des Eqs. (6) la forme de la matrice dynamique dans la nouvelle base:

$$
\left(D_{(\vec{q})}^{\alpha, \beta}-\omega^{2} \delta^{\alpha, \beta}\right) \mathrm{U}_{\overrightarrow{\mathrm{q}}}^{\beta}=0
$$

les vecteurs propres sont caractérisés par $\vec{q}$. On peut étudier cette matrice au voisinage de $\vec{q}=0$.

- $D_{(\overline{\mathrm{q}}=0)}^{\alpha, \beta}=0$, à cause de l'invariance par translation (Eq. (5)).

-si on développe $D_{(\vec{q})}^{\alpha, \beta}$ en $\vec{q}$, les termes linéaires sont nuls parce le potentiel est pair en $\left(\vec{R}_{\vec{L}}-\vec{R}_{\vec{L}^{\prime}}\right)$; les premiers termes non nuls sont quadratiques en $q$ et les valeurs propres de (7) sont, au voisinage de $\vec{q}=0$, de la forme:

$$
\omega^{2}=\mathrm{v}^{2}(\hat{\mathrm{q}}) \mathrm{q}^{2}
$$

les trois vitesses du son ne dépendant que de la direction $\hat{\mathrm{q}}$. Le "modèle de Debye" consiste à négliger, dans la matrice dynamique, les termes en q d'ordre supérieur à deux ainsi que la dépendance en $\hat{\mathrm{q}}$ de cette matrice. Dans cette approximation, la densité d'états, nombre de vecteurs $\overrightarrow{\mathrm{q}}$ auxquels correspond la fréquence $\omega$, est proportionnel à $\omega^{2}$.

\section{1. 3 Etude du cas désordonné}

On peut, a priori, faire le même changement de variables que celui réalisé dans les Eqs. (6), mais on ne diagonalise plus ainsi la matrice dynamique. Celle-ci s'écrit:

$$
\left(D_{\bar{q}^{\prime}, \bar{q}^{\prime}}^{\alpha, \beta}-\omega^{2} \delta_{\alpha, \beta} \delta_{\bar{q}, \bar{q}^{\prime}}\right) U_{\bar{q}^{\prime}}^{\beta}=0
$$

où la matrice $\mathbf{D}$ dépend de $\overrightarrow{\mathrm{q}}$ et de $\overrightarrow{\mathrm{q}}^{\prime}$. Cependant, si $\overrightarrow{\mathrm{q}}$ et $\vec{q}^{\prime}$ tendent tous deux vers zéro, $\mathbf{D}$ ne dépend plus que de $\vec{q}$ car, dans l'espace réel, à grande distance, le potentiel d'interaction entre deux atomes est isotrope et ne dépend que de la distance. On en déduit, dans cette limite, pour les mêmes raisons que dans le cas ordonné, que:

$$
\overrightarrow{\mathrm{q}}, \overrightarrow{\mathrm{q}}^{\prime} \rightarrow 0, \mathrm{D}_{\overrightarrow{\mathrm{q}}, \overrightarrow{\mathrm{q}}^{\prime}}^{\alpha, \beta}=\mathrm{A}_{\gamma, \delta}^{\alpha, \beta} \mathrm{q}_{\gamma} \mathrm{q}_{\delta} .
$$

En outre, l'invariance par translation impose aussi:

$$
\overrightarrow{\mathrm{q}} \text { ou } \overrightarrow{\mathrm{q}}^{\prime} \rightarrow 0, \mathrm{D}_{\overrightarrow{\mathrm{q}}, \mathrm{q}^{\prime}}^{\alpha, \beta}=\mathrm{A}_{\overrightarrow{\mathrm{q}}}^{\alpha, \beta, \gamma} \mathrm{q}^{\gamma} \text {. }
$$

On en déduit que si l'on fait une théorie de perturbation de deuxième ordre, qui revient à replier la matrice dynamique sur un vecteur $\overrightarrow{\mathrm{q}}$ donné, et à négliger le terme en $\omega^{2}$ au dénominateur $\mathrm{du}$ demier terme, la matrice dynamique s'écrit, en négligeant, pour simplifier, les indices de coordonnées cartésiennes $\alpha$ et $\beta$ : 


$$
\omega^{2}-\mathrm{D}_{\overrightarrow{\mathrm{q}}, \overrightarrow{\mathrm{q}}}-\sum_{\overrightarrow{\mathrm{q}}^{\prime}} \frac{\left|\mathrm{D}_{\overrightarrow{\mathrm{q}}, \mathrm{q}^{\prime}}\right|^{2}}{D_{\overrightarrow{\mathrm{q}}^{\prime}, \overrightarrow{\mathrm{q}}^{\prime}}-\mathrm{D}_{\overrightarrow{\mathrm{q}}, \overrightarrow{\mathrm{q}}}}=0
$$

Deux conséquences importantes:

- lorsque $\vec{q} \rightarrow 0$, il y a encore des modes acoustiques: chacun des deux derniers termes de (12) est proportionnel à $q^{2}$ et on peut négliger $D_{\vec{q}, \vec{q}}$ par rapport à $D_{\vec{q}^{\prime}, \vec{q}^{\prime}}$ dans le dénominateur du dernier terme;

- aux fréquences plus élevées, la densité d'états, plus grande pour $|\vec{q}|>|\vec{q}|$, abaisse les fréquences propres puisque, pour la plupart des $\vec{q}^{\prime}$, le dénominateur du dernier terme est positif. C'est ce que l'on voit sur la Figure 1 où est représenté un résultat de Schirmacher et al. [1]: ceux-ci ont calculé les fréquences propres pour un système d'atomes de même masse placés sur un réseau cubique dans lequel on a introduit dans les constantes de force un désordre, caractérisé par la constante $\sigma$; la croissance de $\sigma$ est corrélée avec l'abaissement de la densité d'états. Ce résultat est assez intuitif dans un raisonnement de type perturbatif: la "perturbation" introduite par les termes non diagonaux, $D_{\vec{q}, \vec{q}^{\prime}}$, repousse les états $\vec{q}$ et $\vec{q}^{\prime}$ qu'elle couple; l'effet est surtout visible à basse fréquence où la densité d'états primitive était faible.

\section{1. 4 Etude de la dynamique par diffusion inélastique cohérente de neutrons}

Par cette technique, on mesure directement:

$$
\mathrm{S}_{\ddot{\mathrm{Q}}^{\prime}}(\omega)=\mathrm{FT}\left(\sum_{\tilde{\mathrm{L}}^{\prime}, \mathrm{L}^{\prime}} \mathrm{e}^{\mathrm{i} \overrightarrow{\mathrm{Q}}^{\prime} \cdot\left(\overrightarrow{\mathrm{R}}_{\tilde{\mathrm{L}}^{\prime}}(\mathrm{t})-\overrightarrow{\mathrm{R}}_{\mathrm{L}^{\prime}}(0)\right)}\right)
$$

où $\overrightarrow{\mathrm{Q}}$ est le vecteur de diffusion et FT indique une transformée de Fourier temporelle. Les mesures sont faites habituellement à $\overrightarrow{\mathrm{Q}}$ donné.

Tous les vecteurs propres ont une projection sur $\vec{Q}$ avec des poids différents pour les diverses valeurs propres. On peut formellement écrire le résultat sous la forme:

$$
\mathrm{S}_{\overrightarrow{\mathrm{Q}}}(\omega) \propto \mathrm{g}(\omega) \frac{\mathrm{k}_{\mathrm{B}} \mathrm{T}}{\omega} \sum_{\lambda} \operatorname{Im}\left(\frac{1}{\omega^{2}-\omega_{\lambda}^{2}}\right) \mathrm{P}_{\lambda}(\overrightarrow{\mathrm{Q}})
$$

où $\mathrm{g}(\omega)$ est la densité de modes à la fréquence $\omega$ et $\mathrm{P}_{\lambda}(\overrightarrow{\mathrm{Q}})$ la projection du vecteur propre $\lambda$, normalisé, sur le vecteur $\overrightarrow{\mathrm{Q}}$. Ceci peut s'écrire, approximativement:

$$
\mathrm{S}_{\overline{\mathrm{Q}}}(\omega) \sim \omega^{2} \frac{\mathrm{k}_{\mathrm{B}} \mathrm{T}}{\omega^{2}} \sum_{\lambda} \delta\left(\omega-\omega_{\lambda}\right) \mathrm{P}_{\lambda}(\overrightarrow{\mathrm{Q}}),
$$

en admettant que pour la plus grande partie du spectre, la densité d'état suit encore un modèle de Debye. 11 en résulte un pic à $\omega_{\vec{Q}}$, correspondant à la courbe de dispersion d'un «cristal moyen» pour cette valeur de $\mathrm{Q}$. Souvent les résultats sont présentés sous forme de fonction de corrélation des courants, et non des positions, comme dans (14). Ceci est obtenu en multipliant $\mathrm{S}_{\overrightarrow{\mathrm{Q}}}(\omega)$ par $\omega^{2} / q^{2}$ et a pour résultat pratique de faire apparaître nettement un pic, assez bien marqué pour des valeurs de $\vec{Q}$ pas trop grande par rapport à $2 \pi /$ a (où a est la distance interatomique moyenne), et 


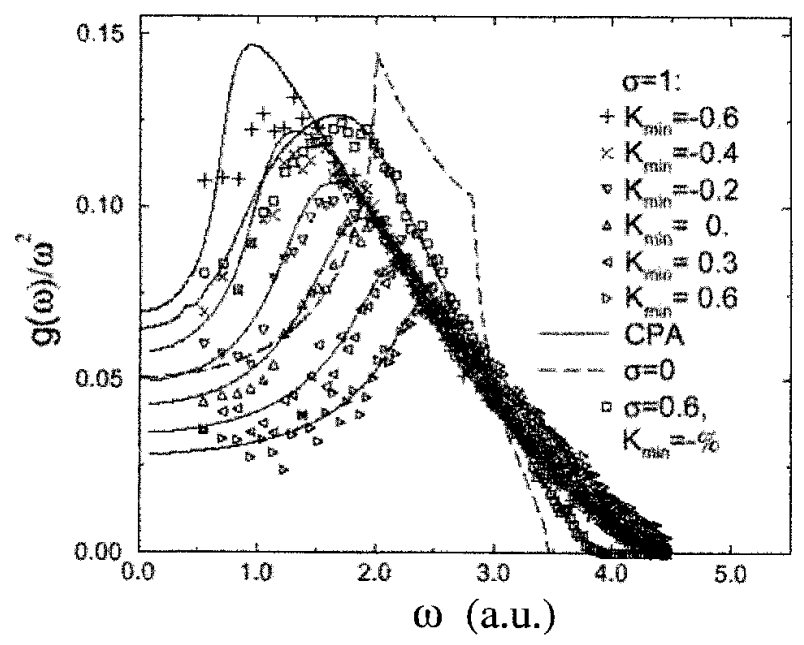

Figure 1. Densité d'états divisée par $\omega^{2}$ pour un cristal cubique avec désordre de constantes de forces, $K_{\vec{L}, \vec{L}^{\prime}}$, entre atomes premiers voisins. Le désordre est caractérisé par une distribution gaussienne des constantes de force, dont le maximum est à $K_{o}=1$, de largeur $\sigma$ avec une coupure basse pour $K_{\min }$. Le maximum de $g(\omega) / \omega^{2}$ se déplace vers les basses fréquences si le désordre croit. D'après Schirmacher et al. [1].

dont la courbe de dispersion en $\overrightarrow{\mathrm{Q}}$ ressemble assez à celle d'un cristal, comme on le voit sur la Figure 2.

- Le "de Gennes Narrowing"

Dans le cristal, on retrouve des phonons acoustiques ( $\omega \sim 0)$ aux pics de Bragg, qui correspondent a des valeurs de $Q=\frac{2 \pi}{a}$. Il restera une trace de cet effet dans un système désordonné: pour cette valeur de $\mathrm{Q}$, il y aura une intensité non négligeable au voisinage de $\omega=0$. Ceci représente la contribution cohérente à l'augmentation importante d'intensité à fréquence nulle que l'on mesure, dans les liquides, pour cette valeur de Q, que l'on appelle le "de Gennes Narrowing" et dont je reparlerai en 4. 1.3.

\subsection{Modes localisés de très basse fréquence}

Il s'agit ici de résultats obtenus par Dynamique Moléculaire.

- on "fabrique" un système désordonné de $\mathrm{N}$ atomes en "refroidissant" très rapidement le liquide correspondant.

- une fois "l'équilibre" obtenu, (chaque atome possède pendant un certain temps une position d'équilibre), on calcule la matrice dynamique, d'où l'on tire les valeurs propres et les vecteurs propres dont on calcule le facteur de participation. Par construction, celui-ci vaut 1 si tous les atomes participent de façon égale à ce vecteur propre et il vaudrait $1 / \mathrm{N}$ si un seul atome était impliqué dans ce vecteur. 

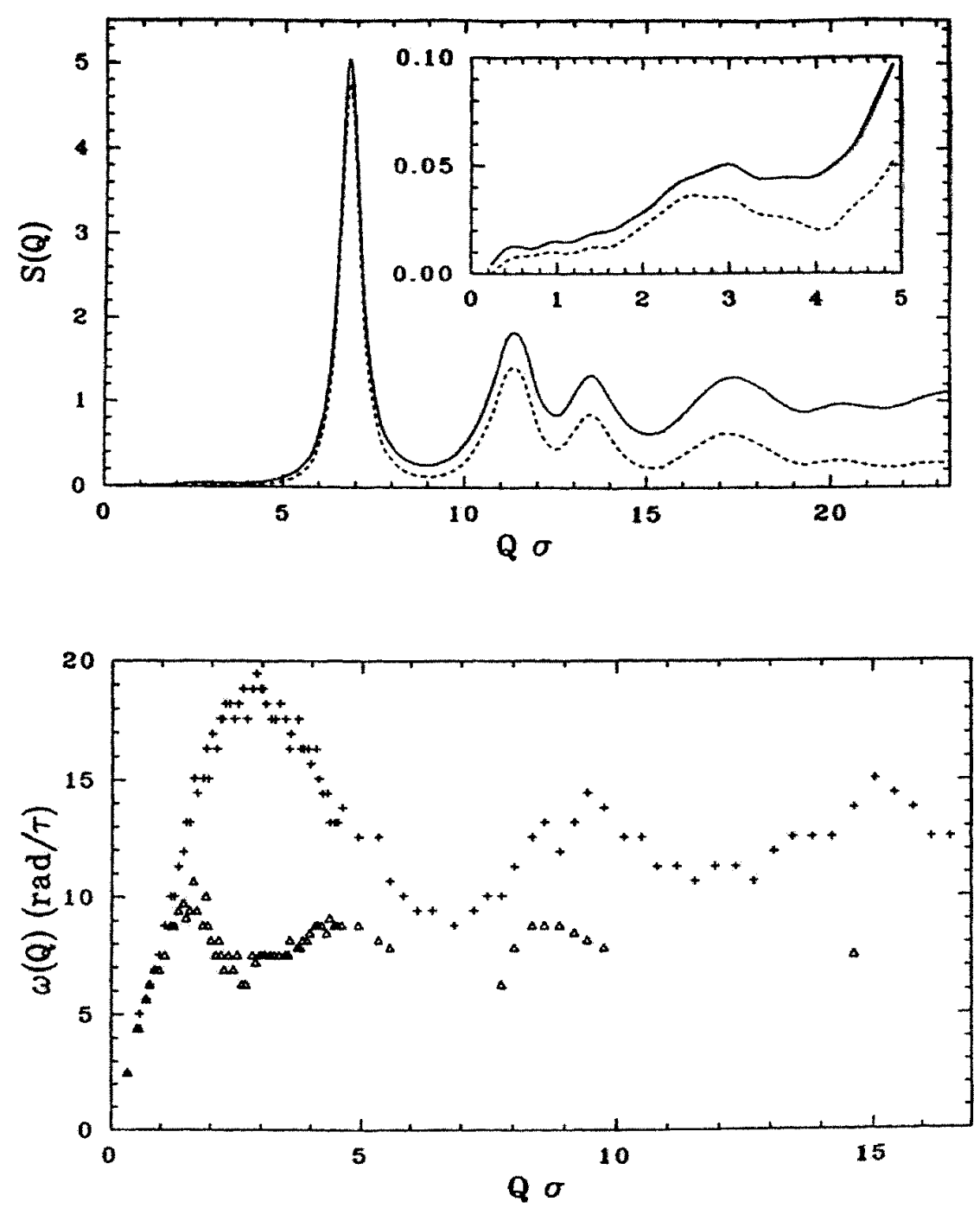

Figure 2. (haut) Facteur de structure d'un modèle de verre métallique. (bas) Courbes de dispersion correspondant, respectivement, au maximum de $S_{\vec{Q}}(\omega)$ (symboles $\Delta$ ) et de $\omega^{2} S(\vec{Q}, \omega)$ (symboles + ) en fonction de $Q \sigma$ où $\sigma$ est le minimum du potentiel interatomique. On note l'existence d'un régime acoustique, d'un maximum pour $Q \sigma \approx \pi$ et d'un minimum correspondant à une fréquence non nulle pour $Q \sigma \approx 2 \pi$. D'après Dzugutov [2]. 
Dans le verre ainsi fabriqué, il existe un petit nombre de modes de très basse fréquence qui ne sont pas acoustiques: ils ne font intervenir que quelques atomes (cf. Figure 3); leur facteur de participation est très petit. Ces modes correspondent à des atomes ayant deux positions possibles d'équilibre et qui sautent de temps en temps de l'une à l'autre. Ces sauts sont corrélés: le saut d'un tel atome entraîne souvent un saut similaire pour un atome voisin qui peut se déplacer à cause du trou laissé par le premier atome et ce mouvement se propage de proche en proche sur quelques atomes.

\subsection{Branches "optiques": Comparaison Ordre Désordre}

\section{2.1 Introduction}

Les systèmes désordonnés ne peuvent que très rarement être reliés à des cristaux monoatomiques; en général, leurs cristaux parents sont polyatomiques. Nous discuterons ici deux cas intéressants pour cette école parce que fréquents: les systèmes moléculaires et iono-moléculaires.

\section{2. 2 Systèmes moléculaires (un seul type de molécules dans le cristal)}

Dans les cristaux dont ils sont issus, il existe, en plus des modes acoustiques, des modes :

- internes à la molécule, généralement d'assez haute fréquence;

- de libration de la molécule;

- (éventuellement) de changement de conformation de cette molécule.

Dans les systèmes désordonnés correspondants:

- La fréquence des modes internes est peu modifiée car leur fréquence est essentiellement liée à des interactions intramoléculaires. La densité d'états est cependant un peu élargie par le désordre: l'interaction avec les molécules voisines modifie légèrement le potentiel vibrationnel, soit directement, soit indirectement par distorsion de la molécule concernée.

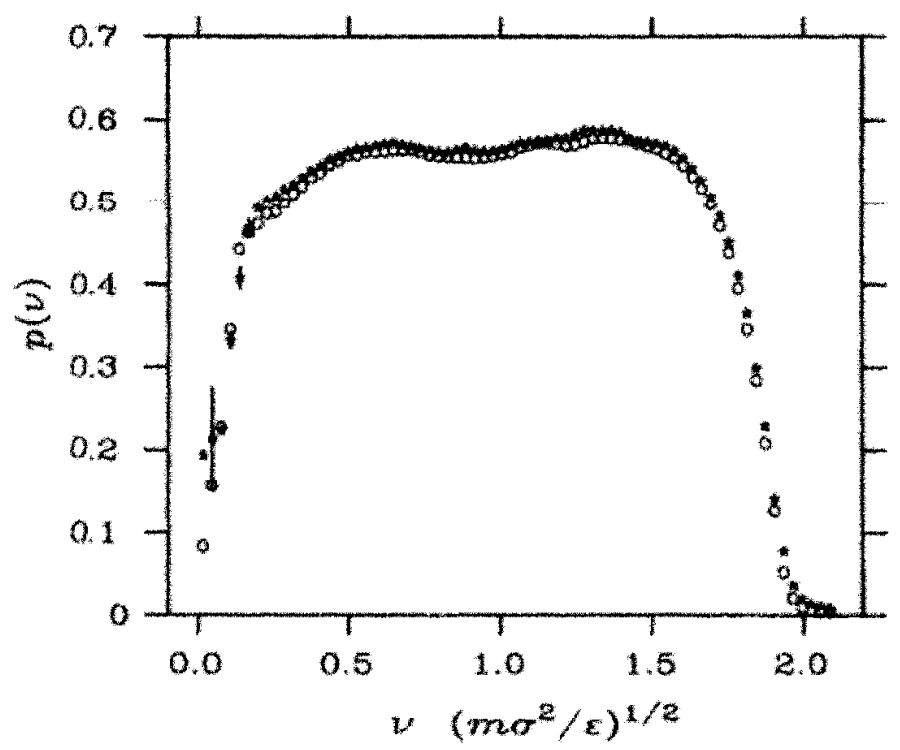

Figure 3. Facteur de participation, $p(\nu)$, relatif aux vibrations de fréquence $\nu$ pour un verre fabriqué à partir d'un potentiel de "sphères molles". On note l'existence de quelques modes de basse fréquence pour lesquels $p(\nu)$ est très faible. D'après Schober et al. [3]. 
- A l'inverse, les deux autres types de modes peuvent être très sensibles au désordre, car ils sont déjà de basses fréquences; l'interaction avec les autres modes tend à les localiser et à augmenter la densité d'états de vibration à basse fréquence. De plus, en diffusion Raman (diffusion inélastique de la lumière), la règle de sélection qui impose de ne détecter, dans les cristaux, que certains modes à $\overrightarrow{\mathrm{q}}=0$ n'est plus valable: tous les modes deviennent actifs et on mesure à basse fréquence une forte densité d'états très large. C'est ce que l'on voit sur la Figure 4 qui compare les spectres Raman de l'ortho-terphényl, cristallisé et sous forme de verre.

\section{2.3 Systèmes iono-covalents}

Ce sont des systèmes dans lesquels les atomes s'assemblent sous forme d'ions très stables, se comportant du point de vue vibrationnel comme des molécules, certains anions situés à la périphérie de l'ion étant communs à deux ou à plusieurs édifices ioniques; la liaison entre les ions est alors très souple cependant que les liaisons à l'intérieur de l'ion sont rigides. Ces types d'assemblages peuvent facilement donner lieu à des verres, et ce sont eux que nous discutons ici. Un exemple classique est le quartz, $\mathrm{S}_{\mathrm{i}} \mathrm{O}_{2}$, formé de tétraèdres $\mathrm{S}_{\mathrm{i}} \mathrm{O}_{4}$ chaque oxygène étant commun à deux tétraèdres; le verre correspondant est la silice. Dans les cristaux de ce type:

- les modes internes des cristaux moléculaires sont remplacés par les vibrations iono-covalentes. - les mouvements relatifs des ions liés par les anions appartenant simultanément à deux entités, sont souvent de très basse fréquence (fréquence nulle si le mouvement relatif de deux entités ne coûte pas d'énergie). On les appelle les "modes de Vallade" [5]. Ils existent, par exemple, dans le quartz.

Ces derniers modes sont très sensibles au désordre et donnent lieu à de fortes densités d'états, assez localisées (voir exposé de M. Foret).

\section{PREMIER EXEMPLE DE DIFFUSION. L'EXEMPLE DES CRISTAUX PLASTIQUES}

La dynamique des systèmes désordonnés se complique beaucoup lorsqu'il n'existe plus de position d'équilibre des constituants. Pour mieux dégager la conséquence (diffusion) de cette absence de position d'équilibre, nous étudions d'abord le cas des cristaux plastiques: ce sont des cristaux (généralement) moléculaires, dont les centres de masse sont ordonnés (ils forment fréquemment un réseau cubique, c.c. ou c.f.c.), mais dont les molécules présentent un désordre orientationnel dynamique. L'étude de cette dynamique orientationnelle est une première approche du problème de la diffusion.

Ex: Néopentane, $\mathrm{C}\left[\mathrm{CH}_{3}\right]_{4}$ : les centres de masse des molécules forment, pour $140 \mathrm{~K}<\mathrm{T}<256 \mathrm{~K}$, un réseau c.f.c. mais les molécules présentent un désordre d'orientation: un des trois axes d'ordre $\overline{4}$ de la molécule est approximativement confondu avec un axe d'ordre $4 \mathrm{du}$ cristal, ẑ par exemple; dans ce cas, les deux autres axes d'ordre $\overline{4}$ sont au voisinage des directions $\hat{x}+\hat{y}$ et $\hat{x}-\hat{y}$ du cristal, conduisant à deux orientations moléculaires différentes. La molécule possède ainsi six orientations privilégiées distinctes, et passe continuellement d'une d'elle aux autres.

\section{3-1 Diffusion et Librations}

Les molécules librent dans leur puits orientationnel puis elles sautent (diffusent) d'un puits à un autre: cette dynamique évolue avec la température, le passage d'un puits à l'autre devenant de plus en plus facile avec l'augmentation de la température. 

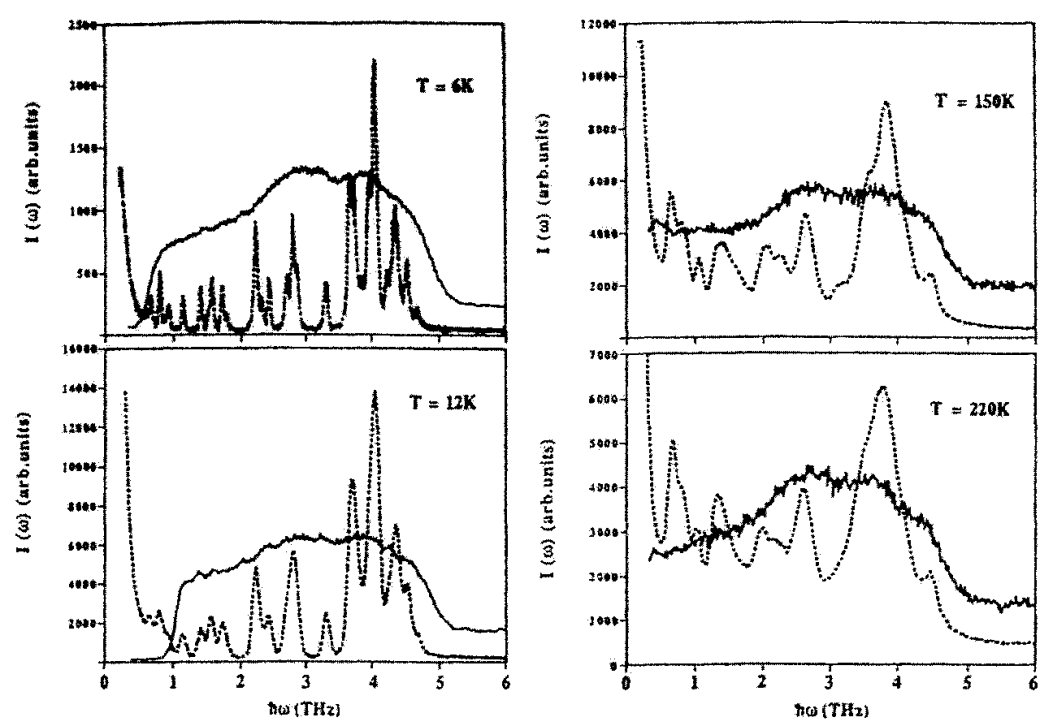

Figure 4. Ortho-terphényl. Comparaison entre les spectres Raman basse fréquence du cristal $\left(T_{\mathrm{f}}=329 \mathrm{~K}\right)$ et du verre correspondant à diverses températures. Les nombreuses raies du cristal, correspondant à des combinaisons de mouvements de translation, de libration, et à des mouvements relatifs des noyaux benzéniques de chaque molécule contrastent avec la très large densité d'états, à peine structurée, du verre. D'après Criado et al. [4].

\section{3-2 Apport de la dynamique moléculaire}

Pour caractériser proprement les divers aspects de cette dynamique (combien de temps la molécule reste-t-elle à l'intérieur d'un puits ? a-t-elle la même probabilité d'atteindre les cinq autres puits? etc....), on réalise d'abord une dynamique moléculaire classique à partir de potentiels interatomiques entre atomes des molécules voisines. On analyse ensuite la dynamique orientationelle individuelle en calculant la fonction de corrélation de fonctions algébriques liées à l'orientation moléculaire spécialement construites pour caractériser le mouvement dont on cherche la dynamique [6]. Par exemple, la Figure 5 montre les résultats pour deux fonctions particulières. La première (Figure 5a) est relative au temps de résidence dans un puits, qui a une décroissance raisonnablement exponentielle. La deuxième (Figure $5 \mathrm{~b}$ ) décrit les changements d'orientation à l'intérieur d'un puits, qui sont nettement plus complexes: à basse température, la molécule simultanément libre dans son puits (c'est l'origine du maximum secondaire vers $40 \mathrm{~cm}^{-1}$ ), alors que les directions des trois axes $\overline{4}$ de la molécule varient d'une façon continue mais lente (diffusion): c'est l'origine du pic central. A plus haute température, cette diffusion devient trop rapide pour rester bien visible alors que la libration reste nettement discernable 


\section{3-3 Diffusion de neutrons incohérente inélastique}

Celle-ci, qui est sensible au mouvement individuels des atomes d'hydrogène, permet, en principe, de déterminer des informations analogues en jouant sur la direction du vecteur $\overrightarrow{\mathrm{Q}}$. En pratique, l'analyse est beaucoup plus compliquée que par Dynamique Moléculaire, en particulier à cause de la forte contribution des mouvements des centres de masse, mais elle donne accès à des valeurs de $\overrightarrow{\mathrm{Q}}$ et $\omega$ inaccessibles par la technique précédente. Il faut, en général, jumeler les deux techniques pour bien analyser cette dynamique.

\section{LIQUIDES - VIBRATIONS ET DIFFUSION}

\section{1 Introduction - les liquides habituels ou "normaux"}

\section{1. 1 Les temps caractéristiques}

Dans un liquide "normal", une particule (atome, molécule) vibre dans son environnement pendant un temps $\tau \sim 10^{-12} \mathrm{~s}$. Les mouvements relatifs de cet environnement permettent l'ouverture de la "cage" dans laquelle la particule est enfermée: celle-ci diffuse (franchit une distance de l'ordre dune distance inter-atomique) en un temps $\tilde{\tau} \approx \tau$.

\section{1. 2 Modes de haute fréquence (modes moléculaires internes ou liaisons iono-covalentes)}

Ces modes sont encore élargis par rapport au cas des systèmes désordonnés car à l'effet statique discuté en 2. 2.2 se superpose un effet dynamique: l'environnement de chaque molécule varie, donnant un temps de vie fini à ces modes.

\section{1. 3 La dynamique basse fréquence}

Les phénomènes sont encore plus complexes car trois types d'effets se superposent. Les deux premiers sont identiques à ceux de 4.1.2, quoique la variation temporelle de l'environnement joue ici un rôle plus important puisque son temps caractéristique est du même ordre de grandeur que la période des modes considérés. Le troisième effet est la diffusion incohérente (fonction de corrélation entre les positions ou orientations de la même particule); cette diffusion donne une contribution lorentzienne centrée à $\omega=0$ dont la largeur dépend de $\overrightarrow{\mathrm{Q}}$. En particulier, comme la diffusion des centres de masse se fait par des sauts de l'ordre d'une distance inter-atomique, elle 

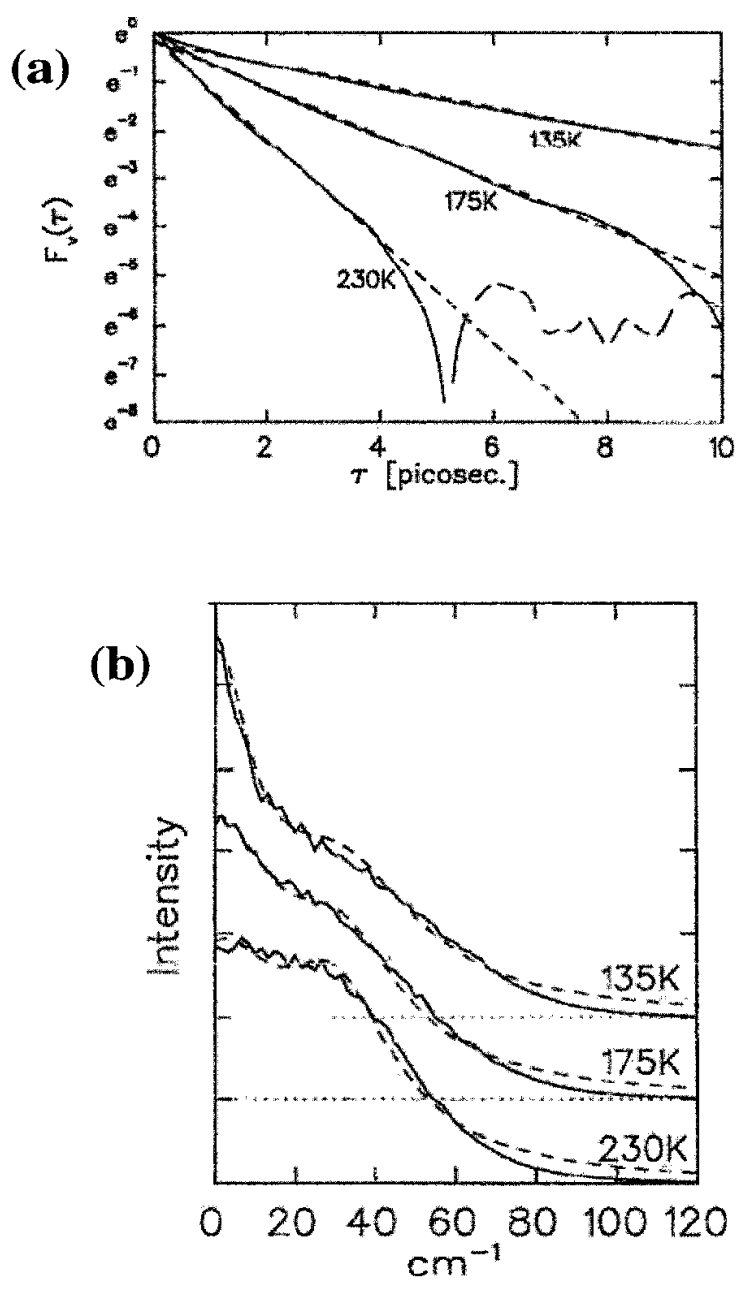

Figure 5. Néopentane, dans sa phase à désordre d'orientation. Fonction d'autocorrélation de fonctions de l'orientation moléculaire caractérisant: (a) la présence de la molécule dans un puits orientationnel donné. (b) la libration autour de l'axe $\hat{z}$ d'une molécule dont l'axe d'ordre $\overline{4}$ est proche de cet axe. Le maximum à $\omega=0$, visible pour $135 \mathrm{~K}$ et $175 \mathrm{~K}$, correspond à la diffusion de l'orientation de la molécule au fond de son puits par suite du changement d'orientation des molécules voisines. D'après Breymann et al. [6]. 
donne une contribution importante aux fonctions de corrélation pour $Q \approx 2 \pi / a$. C'est une deuxième origine du "de Gennes narrowing", intensité très importante au voisinage de $\omega=\mathrm{O}$ pour cette valeur de $Q$, nettement visible sur la Figure 6 , relative au césium à $6^{\circ}$ au dessus de sa température de fusion.

\section{2 Les Liquides surfondus}

\section{2. 1 Introduction}

Un liquide surfondu est un état métastable qui existe, dans certains liquides, pour $T<\mathrm{T}_{\mathrm{f}}$ (température de fusion). $\tau$ (et aussi $\widetilde{\tau}$ ) croit lorsque $\mathrm{T}$ diminue.

Les liquides vitrifiables sont ceux pour lesquels cet état métastable peut se maintenir lorsque $\tau$ tend vers l'infini, et $\tau$ représente aussi le temps nécessaire pour le retour à l'équilibre du liquide surfondu, à la température $\mathrm{T}$, s'il est soumis à une petite perturbation. Le liquide surfondu est dit formé un verre lorsque $\tau$ devient de l'ordre de l'heure.

Un aspect intéressant de la dynamique de ces liquides est le découplage entre l'échelle de temps des vibrations, identique à celle d'un liquide normal, et celle de la diffusion, caractérisée par $\tau$. On peut étudier ainsi beaucoup plus finement cette dernière.

\subsection{Apport de la dynamique moléculaire}

Beaucoup d'études ont été faites ainsi ces dernières années, et ont apporté un très grand nombre de renseignements. Je ne discuterai ici que deux aspects.

\section{2. 2. 1 Modes instantanés normaux. Aspects collectifs de la dynamique}

On réalise une Dynamique Moléculaire du liquide, puis on choisit une configuration instantanée donnée, ce qui définit un jeu de $\vec{R}_{\vec{L}}$ (qui ne sont pas ici des positions d'équilibre). Pour ces $\vec{R}_{\vec{L}}$, on calcule la matrice $D_{\overline{\mathrm{L}}, \overline{\mathrm{L}}^{\prime}}^{\alpha, \beta}$ (c.f. 2. 1) dont les valeurs propres sont appelées Modes Normaux Instantanés. Leur densité d'états comprend des fréquences réelles (modes de vibrations harmoniques qui laissent les atomes au voisinage de $\vec{R}_{\vec{L}}$ ) et des fréquences imaginaires: les vecteurs propres correspondants décrivent des mouvements collectifs qui vont faire évoluer la structure microscopique du liquide. Le nombre des fréquences imaginaires diminue avec la température, ce qui explique que l'état métastable peut se conserver plus longtemps.

\section{2. 2 . 2 Inhomogénéités dynamiques. Aspect partiellement individuel, partiellement collectif de la diffusion.}

Dans l'approximation classique de la diffusion, la distribution des distances, $r(t)$, parcourues par les atomes pendant un temps $t$, multipliee par $r^{2}$, est une gaussienne dont la largeur est proportionnelle à $t^{0.5}$. Dans les liquides surfondus, il existe une échelle de temps intermédiaire entre $10^{-12} \mathrm{~s}$ (temps caractéristique des vibrations normales du liquide) et $\tau$ pour laquelle cette distribution n'est pas gaussienne, ce que l'on caractérise par un facteur de "non gaussianity". Ce dernier a un maximum pour un temps donné (c.f. Figure 7b). On peut identifier les atomes responsables de cet écart à la loi classique: ils forment des ensembles pseudo-lineaires (Figure 7c), qui sont la contrepartie dynamique des modes localisés décrits en 2. 1. 5. Dans ces ensembles, tous les atomes se déplacent successivement, chaque atome remplaçant l'autre; ils sont vraisemblablement le mécanisme principal du retour à l'équilibre du liquide perturbé, donc de la diffusion des particules. 

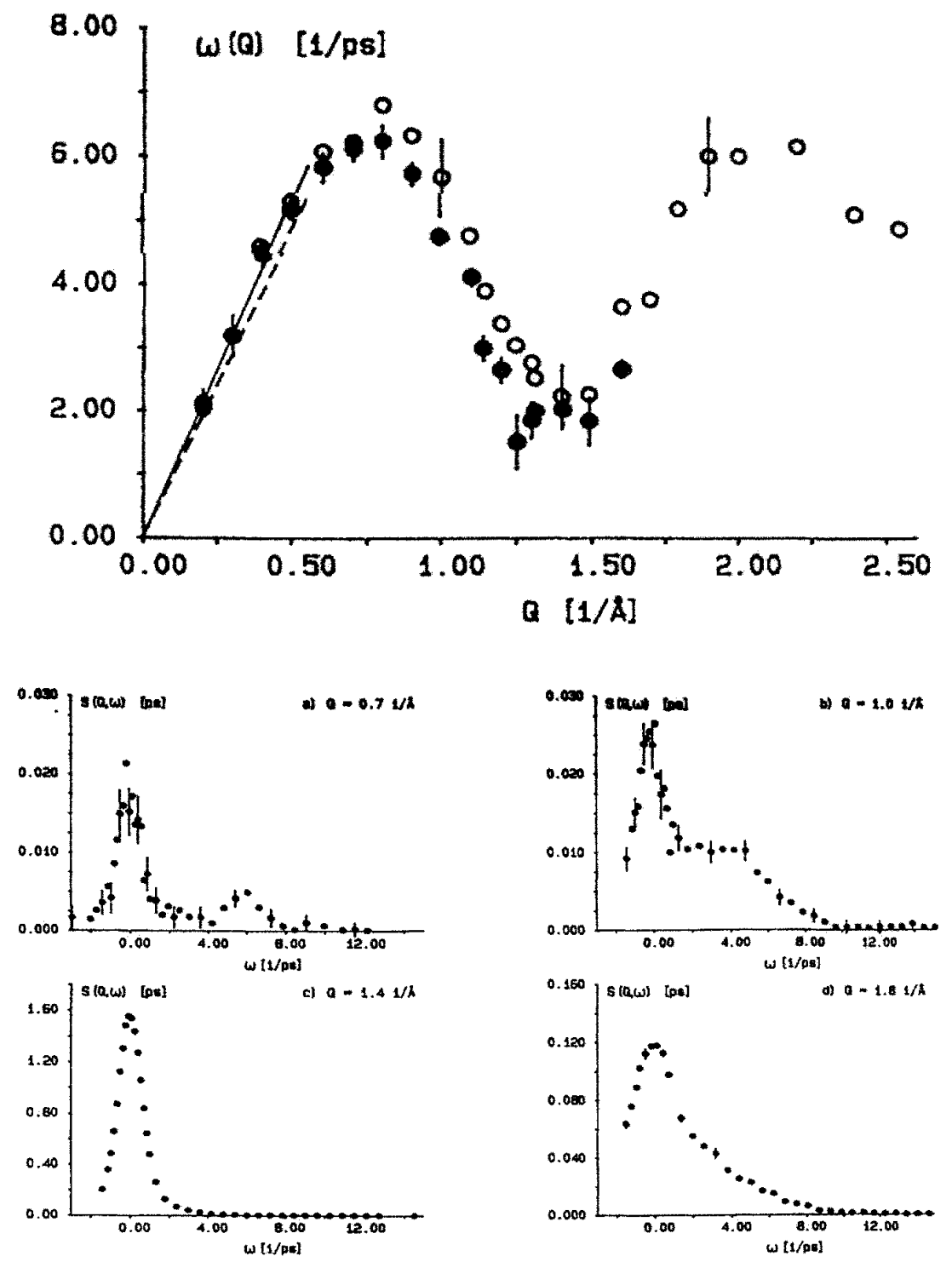

Figure 6. Césium liquide à $308 \mathrm{~K}$. (haut) Courbe de dispersion tracée à partir du maximum secondaire de la fonction de corrélation des courants (c.f. Figure 2 (bas)). (bas) Fonction de corrélation des positions pour différentes valeurs de $Q$. Le spectre pour $Q \approx 2 \pi / a$ n'a de maximum que pour $\omega=0$ et une intensité beaucoup plus grande que pour les autres valeurs de $Q$. D'après Bodensteiner et al. [7]. 


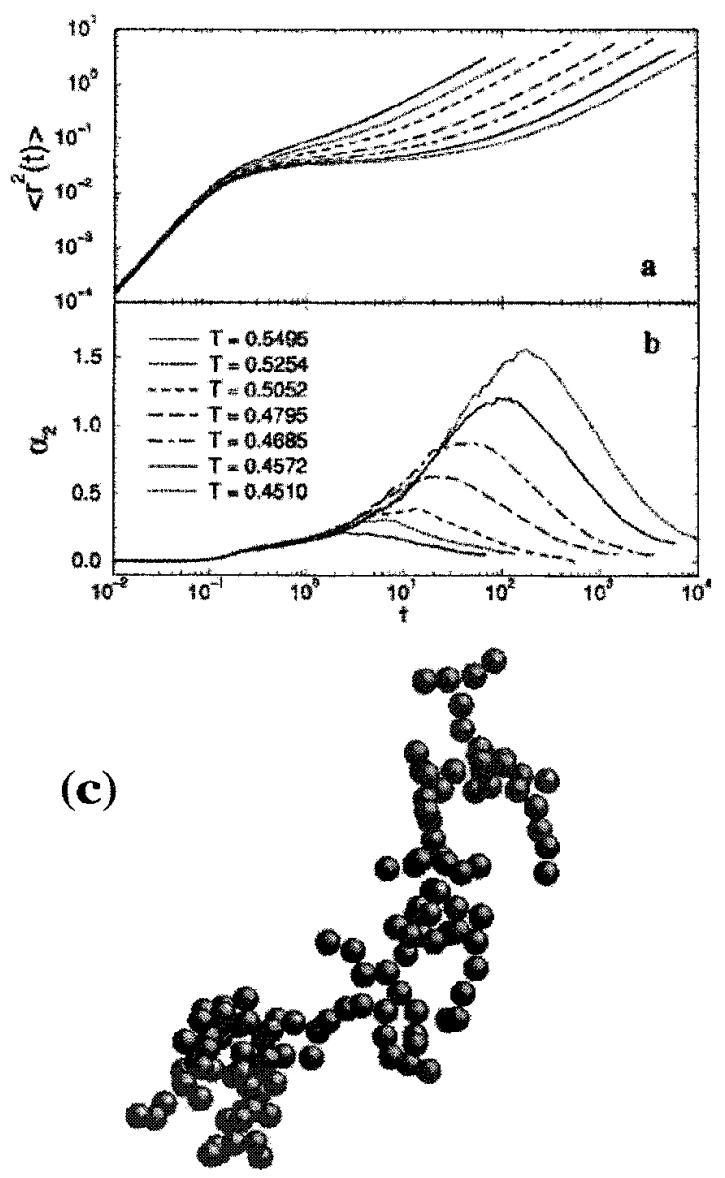

Figure 7. Hétérogénéités dynamiques détectées par Dynamique Moléculaire dans un liquide surfondu. (a) valeur moyenne du carré de la distance parcourue, pour diverses températures. (b) évolution temporelle, aux mêmes températures, du facteur de "non gaussianity", caractérisant l'existence de particules dont le mouvement est anormalement rapide. (c) ensemble de particules, à $T=0.451$, responsable de l'existence du facteur de "non gaussianity". L'aspect quasi-unidimensionnel de cet ensemble explique le mouvement presque simultané de ces particules. D'après Donati et al. [8].

\section{Références}

1. W. Schirmacher, G. Diezemann, C. Ganter, Phys. Rev. Lett. 81, 136 (1998).

2. M. Dzugutov, Physica A 201, 430 (1993).

3. H.R. Schober and B.B. Laird, Phys. Rev. B 44, 6746 (1991).

4. A. Criado, F.J. Bermejo, A. de Andres, J.L. Martinez, Molecular Physics 82, 787 (1994).

5. M. Vallade, B. Berge and G. Dolino, J. Phys. I France 2, 1481 (1992).

6. W. Breymann and R.M. Pick, J. Chem. Phys. 100, 2232 (1994).

7. T. Bodensteiner, C. Morkel, P. Müller, W. Gläser, J. non-Cryst. Sol. 117, 116 (1990).

8. C. Donati, S.C. Glotzer, P.H. Poole, W. Kob, S.J. Plimpton, Phys.Rev. E 60, 3107 (1999). 